\title{
A polynomial invariant of finite quandles
}

\author{
Sam Nelson \\ Department of Mathematics, Whittier College \\ 13406 Philadelphia, P.O. Box 634 \\ Whittier, CA 90608-0634 \\ knots@esotericka.org
}

\begin{abstract}
We define a two-variable polynomial invariant of finite quandles. In many cases this invariant completely determines the algebraic structure of the quandle up to isomorphism. We use this polynomial to define a family of link invariants which generalize the quandle counting invariant.
\end{abstract}

KEYWORDS: Finite quandles, link invariants

2000 MSC: 57M27, 176D99

\section{Introduction}

A quandle is an algebraic structure whose axioms are transcriptions of the three Reidemeister moves, making quandles a natural source of invariants of knots and links. In [8], Joyce associated a finitely generated quandle to each tame knot or link $L \subset S^{3}$ and proved that this knot quandle determines the knot type up to orientation-reversing homeomorphism of pairs $\left(L, S^{3}\right)$.

Since then, much work has been done in the study of knot invariants defined in terms of the knot quandle, such as [4], 3], etc. In particular, for any finite quandle $T$ there are invariants of knots and links defined in terms of the set $\operatorname{Hom}(Q(K), T)$ of quandle homomorphisms from the knot quandle $Q(K)$ into a finite target quandle $T$. Since isomorphic target quandles $T$ and $T^{\prime}$ define the same invariants, we would like to have a convenient way of distinguishing finite quandles. Moreover, understanding the structure of these finite quandles can lead to a better understanding of their link invariants.

In this paper we describe a two-variable polynomial invariant of finite quandles. This polynomial encodes a set with multiplicities arising from counting trivial actions of elements on other elements; the polynomial is a convenient way to encode this information with the added benefit of obtaining integer-valued invariants via specialization of $s$ and $t$. While not a complete invariant, this polynomial does seem to be quite good at distinguishing finite quandles. Indeed, in some cases the invariant completely determines the structure of the quandle.

One motivation for this construction comes from consideration of the nature of identity elements and kernels of homomorphisms. Specifically, groups have a unique identity element which acts trivially on the rest of the group, and the preimage of this element under a homomorphism measures how much information about the domain group is lost in the homomorphism. Unlike groups, quandles do not have a unique identity element; rather, every element in a quandle acts trivially on itself and possibly some other elements. We might say that in a group, the "trivial action" is concentrated in a single element, while in a quandle it is distributed throughout the set. Our polynomial may then be understood as a way of quantifying how this "trivial action" is distributed.

The paper is organized as follows. In section 2, we recall the definition of a quandle and some examples of quandles. We then define the $q p$ polynomial and list some examples. In section 3, we define a generalization of $q p$ for subquandles of a larger quandle and a $q p$-based Laurent polynomial 
associated to a homomorphism between finite quandles. We the use $q p$ to define a family of link invariants which are jazzed-up versions of the familiar quandle counting invariant. In section 4, we list some questions for future research and end with a table listing the $q p$ values for all quandles with order up to 5 .

\section{Quandles and a polynomial invariant}

We begin with a definition from Joyce, $[8]$.

Definition 1 A quandle is a set $Q$ with a binary operation $\triangleright: Q \times Q \rightarrow Q$ satisfying

(i) for every $a \in Q, a \triangleright a=a$,

(ii) for every $a, b \in Q$, there is a unique $c \in Q$ such that $a=c \triangleright b$, and

(iii) for every $a, b, c \in Q,(a \triangleright b) \triangleright c=(a \triangleright c) \triangleright(b \triangleright c)$.

If (ii) and (iii) are satisfied, then $Q$ is a rack [5]; if (iii) is satisfied, then $Q$ is a shelf [1].

The axioms can be understood as transcriptions of the three Reidemeister moves where each arc in the knot diagram corresponds to a quandle element and the quandle operation corresponds to an arc going through a crossing. Some well-known examples of quandles include:

Example 1 A group $G$ is a quandle under various operations, such as

1. $x \triangleright y=y^{-1} x y$, called a conjugation quandle,

2. $x \triangleright y=y^{-n} x y^{n}$, an $n$-fold conjugation quandle,

3. $x \triangleright y=s\left(x y^{-1}\right) y$ where $s \in \operatorname{Aut}(\mathrm{G})$, called a homogeneous quandle.

Example 2 A commutative ring $R$ with a choice of invertible element $t \in R$ is a quandle under

$$
x \triangleright y=t x+(1-t) y .
$$

Indeed, it's easy to see that this is only possible quandle structure on $R$ with the quandle operation defined as a linear polynomial: set $x \triangleright y=A x+B y+C$. Then axiom (i) requires

$$
x=A x+B x+C=(A+B) x+C
$$

for all $x \in R$, so comparing coefficients we have $C=0$ and $B=1-A$. More generally, we may replace the ring $R$ with a module over $\mathbb{Z}\left[t^{ \pm 1}\right]$; quandles of this type are called Alexander quandles. See [10] for more.

Example 3 Let $R$ be a commutative ring, $M$ an $R$-module and $\langle\rangle:, M \times M \rightarrow R$ an anti-symmetric bilinear form. Then $M$ is a quandle under

$$
x \triangleright y=x+\langle x, y\rangle y .
$$

If $R$ is a field and $\langle$,$\rangle is non-degenerate, then we call M$ a symplectic quandle. See [12] and 9] for more.

Let $Q=\left\{x_{1}, x_{2}, \ldots, x_{n}\right\}$ be a finite quandle. We can describe the algebraic structure of $Q$ with an $n \times n$ matrix $M_{Q}$ satisfying $M_{Q}[i, j]=k$ where $x_{i} \triangleright x_{j}=k$. In other words, the matrix of $Q$ is just the quandle operation table without the " $x$ "s. This quandle matrix notation is convenient for symbolic computation purposes [7], [6]. 
Definition 2 Let $Q$ be a finite quandle. For any element $x \in Q$, let

$$
c(x)=|\{y \in Q: y \triangleright x=y\}| \quad \text { and let } r(x)=|\{y \in Q: x \triangleright y=x\}| .
$$

Then we define the quandle polynomial of $Q, q p_{Q}(s, t)$, to be $q p_{Q}(s, t)=\sum_{x \in Q} s^{r(x)} t^{c(x)}$. We will refer to $r(x)$ and $c(x)$ as the row count and column count of $x$ respectively.

That is, $r(x)$ is the number of elements of $Q$ which act trivially on $x$, while $c(x)$ is the number of elements of $Q$ on which $x$ acts trivially. These numbers are easily computed from the matrix of $Q$, simply by going through rows and columns and counting occurrences of the row number. Note that if $Q$ has infinite cardinality, then $c(x)$ and $r(x)$ may not be finite and $q p_{Q}(s, t)$ may not be defined.

Proposition 1 If $Q$ and $Q^{\prime}$ are isomorphic finite quandles, then $q p_{Q}(s, t)=q p_{Q^{\prime}}(s, t)$.

Proof. Suppose $f: Q \rightarrow Q^{\prime}$ is an isomorphism of quandles and fix $x \in Q$. Then for every $y \in C(x)=\{y \in Q: y \triangleright x=y\}$, we have $f(y) \triangleright f(x)=f(y \triangleright x)=f(y)$, so $f(y) \in C(f(x))$ and we have $|C(x)| \leq \mid C\left(f(x) \mid\right.$. Repeating the same argument with $f^{-1}$ yields the opposite inequality, and we have $c(x)=c(f(x))$. A similar argument shows that $r(x)=r(f(x))$. Then we have

$$
q p_{Q}(s, t)=\sum_{x \in Q} s^{r(x)} t^{c(x)}=\sum_{f(x) \in Q^{\prime}} s^{r(x)} t^{c(x)}=\sum_{f(x) \in Q^{\prime}} s^{r(f(x))} t^{c(f(x))}=q p_{Q^{\prime}}(s, t) .
$$

Definition 3 The specialization $q p_{Q}(1, t)$ is the column polynomial of $Q$. The specialization $q p_{Q}(s, 1)$ is the row polynomial of $Q$.

Example 4 The trivial quandle of order $n, T_{n}$, satisfies $x_{i} \triangleright x_{j}=x_{i}$ for all $i, j \in\{1,2, \ldots, n\}$. It has quandle matrix

$$
M_{T_{n}}=\left[\begin{array}{cccc}
1 & 1 & \ldots & 1 \\
2 & 2 & \ldots & 2 \\
\vdots & \vdots & & \vdots \\
n & n & \ldots & n
\end{array}\right] \quad \text { and } \quad q p_{T_{n}}(s, t)=n s^{n} t^{n} .
$$

Proposition 2 Evaluating $q p_{Q}(1,1)$ yields $|Q|$, the cardinality of $Q$.

Proof.

$$
q p(1,1)=\sum_{x \in Q} 1^{r(x)} 1^{t(x)}=\sum_{x \in Q} 1=|Q|
$$

Thus, we may regard $q p_{Q}(s, t)$ as a kind of enhanced version of cardinality for finite quandles. It's easy to see that $q p_{Q}(s, t)$ completely determines the quandle structure for some quandles, such as $T_{n}$ above. In fact, $q p$ distinguishes all quandles of order 3 and 4 , as shown in table 1 .

Example 5 There is only one possible quandle $Q$ with $q p_{Q}(s, t)=3 s t$. To see this, consider the matrix of a quandle $Q$ with $q p_{Q}(s, t)=3 s t$. This is a $3 \times 3$ quandle matrix such that every row and column of $M_{Q}$ has precisely one entry equal to its row number. We know (see [7]) that every quandle matrix must have columns which are permutations of $\{1,2, \ldots, n\}$ and must have entries 
$1,2, \ldots, n$ along the diagonal. Then we must have $M_{Q}=\left[\begin{array}{lll}1 & 0 & 0 \\ 0 & 2 & 0 \\ 0 & 0 & 3\end{array}\right]$ (here we use " 0 " as a blank to be filled in). Now, the $(2,1)$ entry cannot be 2 , or the element $x_{1} \in Q$ would have $c\left(x_{1}\right)>1$, and similarly the $(3,1)$ entry must be 2 . Indeed, just the $c(x)$ numbers here are enough to determine that we must have $M_{Q}=\left[\begin{array}{lll}1 & 3 & 2 \\ 3 & 2 & 1 \\ 2 & 1 & 3\end{array}\right]$.

There are examples of non-isomorphic quandles with the same $q p$ values, as the next example shows.

Example 6 The Alexander quandles $\mathbb{Z}_{5}\left[t^{ \pm 1}\right] /(t-2), \mathbb{Z}_{5}\left[t^{ \pm 1}\right] /(t-3)$ and $\mathbb{Z}_{5}\left[t^{ \pm 1}\right] /(t-4)$ all have $q p_{Q}(s, t)=5 s t$; the fact that they are non-isomorphic follows from corollary 2.2 in [10].

The $q p$ polynomial distinguishes between some quandles which have the same orbit decomposition but different structure maps (see [11]).

Example 7 The quandles with quandle matrices

$$
M_{Q}=\left[\begin{array}{llllll}
1 & 3 & 2 & 1 & 1 & 1 \\
3 & 2 & 1 & 2 & 2 & 2 \\
2 & 1 & 3 & 3 & 3 & 3 \\
4 & 4 & 4 & 4 & 6 & 5 \\
5 & 5 & 5 & 6 & 5 & 4 \\
6 & 6 & 6 & 5 & 4 & 6
\end{array}\right] \quad \text { and } \quad M_{Q^{\prime}}=\left[\begin{array}{llllll}
1 & 3 & 2 & 1 & 3 & 2 \\
3 & 2 & 1 & 3 & 2 & 1 \\
2 & 1 & 3 & 2 & 1 & 3 \\
4 & 6 & 5 & 4 & 6 & 5 \\
6 & 5 & 4 & 6 & 5 & 4 \\
5 & 4 & 6 & 5 & 4 & 6
\end{array}\right]
$$

have $q p_{Q}(s, t)=6 s^{4} t^{4}$ and $q p_{Q^{\prime}}(s, t)=6 s^{2} t^{2}$ respectively. Both have the same orbit decomposition, namely two orbits isomorphic to the Alexander quandle $\mathbb{Z}_{3}\left[t^{ \pm 1}\right] /(t-2)$, but the two have different structure maps.

Definition 4 A quandle is connected if it has only one orbit. A quandle is Latin or strongly connected if the quandle operation is left-invertible, in addition to the right-invertibility required by axiom (ii) 1 In particular, the rows of a Latin quandle are also permutations of $\{1,2, \ldots, n\}$

Proposition 3 A Latin quandle $Q$ satisfies $q p_{Q}(s, t)=|Q|$ st.

Proof. Let $Q$ be a Latin quandle and fix an element $x_{i}$. Since $Q$ is a quandle, the $(i, i)$ entry of $M_{Q}$ is $i$, so $c\left(x_{i}\right) \geq 1$ and $r\left(x_{i}\right) \geq 1$. Now, suppose $r\left(x_{i}\right)>1$. Then there is a column $j \neq i$ with $M_{Q}[i, j]=i$. But then row $i$ is not a permutation of $\{1,2, \ldots, n\}$, contradicting the fact that $Q$ is Latin. Hence, we must have $r\left(x_{i}\right)=1$.

Similarly, if $c\left(x_{i}\right)>1$ then there is some $j \neq i$ such that $M_{Q}[j, i]=j$, and then we have $r\left(x_{j}\right)>1$, contradicting our conclusion above. Hence $c\left(x_{i}\right)=1$. Since $i$ was arbitrary, we have

$$
q p_{Q}(s, t)=\sum_{x \in Q} s t=|Q| s t .
$$

Direct computations show that the converse of proposition 2 has no counterexamples with cardinality less than 7 . Thus we have:

Conjecture 1 Every quandle $Q$ with $q p_{Q}(s, t)=|Q|$ st is Latin.

\footnotetext{
${ }^{1}$ Latin quandles are a type of distributive quasigroup.
} 
We may also define the $q p$ invariant for non-quandle racks; such racks may have $q p_{R}(s, t)=0$, as in the next example.

Example 8 Let $R=\mathbb{Z}_{n}$ with $i \triangleright j=i+k$ for a fixed $k \in R$. Then $R$ is a rack since

$$
(x \triangleright y) \triangleright z=(x+k) \triangleright z=x+2 k
$$

while

$$
(x \triangleright z) \triangleright(y \triangleright z)=(x+k) \triangleright(y+k)=x+2 k .
$$

The matrix of $R$ has every entry in row $i$ equal to $i+k$, so if $k \neq 0 \in \mathbb{Z}_{n}$, then $c(x)=r(x)=0$ for all $x \in R$, and $q p_{R}(s, t)=0$.

Indeed, if $q p_{R}(1,1) \neq|R|$ for a rack $R$, then $R$ is not a quandle.

\section{Subquandles, homomorphisms and a family of link invariants}

In this section we define a generalization of $q p_{Q}(s, t)$ which gives information about how a subquandle is embedded in a larger quandle and we use this to define a family of link invariants.

Definition 5 Let $S \subset Q$ be a subquandle of $Q$. The subquandle polynomial $q p_{S \subset Q}(s, t)$ of $S \subset Q$ is

$$
q p_{S \subset Q}(s, t)=\sum_{x \in S} s^{r(x)} t^{c(x)}
$$

where $r(x)$ and $c(x)$ are the same as in definition 2

Example 9 Let $Q$ be the quandle with matrix $M_{Q}=\left[\begin{array}{llll}1 & 1 & 2 & 2 \\ 2 & 2 & 1 & 1 \\ 3 & 3 & 3 & 3 \\ 4 & 4 & 4 & 4\end{array}\right]$ and let $S=\{1,2\}$ and $S^{\prime}=\{3,4\}$. Then both $S$ and $S^{\prime}$ are isomorphic to $T_{2}$, the trivial quandle of order 2 , but they are embedded in $S$ in a different way; this is reflected in their subquandle polynomial values $q p_{S \subset Q}(s, t)=2 s^{2} t^{4}$ and $q p_{S^{\prime} \subset Q}(s, t)=2 s^{4} t^{2}$.

Proposition 4 If a finite quandle $Q=Q_{1} \cup Q_{2} \cup \cdots \cup Q_{n}$ is a disjoint union of several subquandles (e.g., the orbit subquandles) then we have

$$
q p_{Q}(s, t)=\sum_{i=1}^{n} q p_{Q_{i} \subset Q}(s, t)
$$

Proof.

Definition 6 Let $f: Q \rightarrow Q^{\prime}$ be a homomorphism of finite quandles. Let $K_{q p}(f) \in \mathbb{Z}\left[s^{ \pm 1}, t^{ \pm 1}\right]$ be given by

$$
K_{q p}(f)=\sum_{x \in Q} s^{r(f(x))-r(x)} t^{c(f(x))-c(x)} .
$$

Proposition 5 If $f: Q \rightarrow Q^{\prime}$ is injective, then every exponent in $K_{q p}(f)$ is nonnegative; if $f$ is surjective, then every exponent in $K_{q p}(f)$ is nonpositive. If $f$ is an isomorphism, then $K_{q p}(f)=|Q|$.

Proof. This is similar to the proof of proposition 1,

Thus, $K_{q p}(f)$ has a philosophical similarity to the kernel of a homomorphism. Note, however, that the converse of proposition 5 is not true, as the next example shows. 
Example 10 Let $f: T_{2} \rightarrow T_{3}$ be the constant map $f(1)=f(2)=1 \in T_{3}$ where $T_{n}$ is the trivial quandle of order $n$. Then we have $K_{q p}(f)=2 s^{3-2} t^{3-2}=2 s t$, though $f$ is not injective.

Recall that for any knot or link $K$, there is an associated knot quandle $Q(K)$, and that for a given finite quandle $T$ the set of quandle homomorphisms

$$
\operatorname{Hom}(Q(K), T)=\{f: Q(K) \rightarrow T: f(x \triangleright y)=f(x) \triangleright f(y)\}
$$

is a source of computable knot invariants. Specifically, we can take the cardinality of the set, which gives us an integer-valued invariant. Alternatively, we can count the homomorphisms weighted by a cocycle in one of the various quandle cohomology theories (described in [3], 2] etc.); these cocycles provide a way of squeezing extra information out of the set of homomorphisms. We can use the subquandle polynomials of the image of each homomorphism in a similar way 2

Definition 7 Let $K$ be a link and $T$ a finite quandle. Then for every $f \in \operatorname{Hom}(Q(K), T)$, the image of $f$ is a subquandle of $T$. Define the subquandle polynomial invariant $\Phi_{q p}(K)$ to be the set with multiplicities

$$
\Phi_{q p}(K)=\left\{q p_{\operatorname{Im}(f) \subset T}(s, t): f \in \operatorname{Hom}(Q(K), T)\right\} .
$$

Normally, we encode sets with multiplicities whose elements are integers as polynomials where the multiplicities appear as coefficients and the elements of the set are powers of a variable; in this case, however, the elements of the set are already polynomials, and polynomials with polynomial powers seem a little awkward. However, we can derive convenient polynomial-valued specializations of $\Phi_{q p}(K)$ by choosing values of $s$ and $t$ in $\mathbb{Z}$ and evaluating.

Definition 8 Let $K$ be a link, $T$ a finite quandle, $s_{0}, t_{0} \in \mathbb{Z}$. Define the specialized subquandle polynomial invariant $\Phi_{q p}\left(K, s_{0}, t_{0}\right) \in \mathbb{Z}\left[z^{ \pm 1}\right]$ to be the polynomial

$$
\Phi_{q p}\left(K, s_{0}, t_{0}\right)=\sum_{f \in \operatorname{Hom}(Q(K), T)} z^{q p_{\operatorname{Im}(f) \subset T}\left(s_{0}, t_{0}\right)} .
$$

Example 11 If we specialize $s_{0}=t_{0}=0$, then we have

$$
\left.\left.\Phi_{q p}(K, 0,0)=\sum_{f \in \operatorname{Hom}(Q(K), T)} z^{0}=\mid \operatorname{Hom}(Q) K\right), T\right) \mid,
$$

so the specializations of $\Phi_{q p}(K)$ are generalizations of the quandle counting invariant.

In general, specializations of $\Phi_{q p}(K)$ contain more information than the unadorned counting invariant, as the next example shows.

Example 12 The links $L_{1}$ and $L_{2}$ have quandle counting invariant

$$
\left|\operatorname{Hom}\left(Q\left(L_{1}\right), T\right)\right|=\left|\operatorname{Hom}\left(Q\left(L_{2}\right), T\right)\right|=13
$$

with the quandle $T$ with quandle matrix listed below. However, the specialized quandle polynomials

$$
\Phi_{q p}\left(L_{1}, 1,1\right)=5 z+2 z^{2}+6 z^{3} \quad \text { and } \quad \Phi_{q p}\left(L_{2}, 1,1\right)=5 z+2 z^{2}+6 z^{5}
$$

distinguish the links.
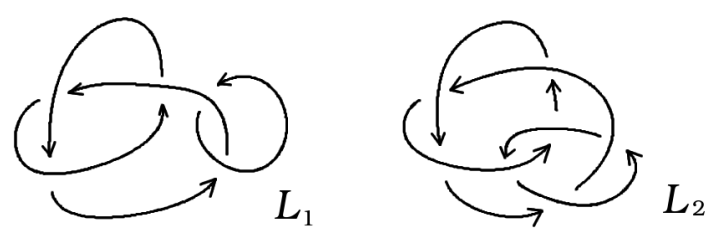

$$
M_{T}=\left[\begin{array}{lllll}
1 & 3 & 2 & 3 & 2 \\
3 & 2 & 1 & 1 & 3 \\
2 & 1 & 3 & 2 & 1 \\
5 & 5 & 5 & 4 & 4 \\
4 & 4 & 4 & 5 & 5
\end{array}\right]
$$

\footnotetext{
${ }^{2}$ While it might seem more natural to count $K_{q p}(f)$ for each quandle homomorphism $f$, in general $q p$ is not well-defined for knot quandles, which are infinite. If we set $r(x)=c(x)=0$ for infinite quandles, then counting $K_{q p}(f)$ yields the listed definition.
} 


\section{Questions for further research}

In this section we list a few questions whose answers may prove interesting.

Under what conditions are two finite quandles $q p$-equivalent? What information in addition to $q p_{Q}(s, t)$ must be specified in order to fully determine the quandle type of $Q$ up to isomorphism? For which polynomials $p \in \mathbb{Z}[s, t]$ is the set of quandles $Q$ with $q p_{Q}(s, t)=p$ nonempty? It's easy to see that such a $p$ must have all positive coefficients whose sum is $|Q|$ and each term must have degree $d$ satisfying $2 \leq d \leq 2|Q|$ (or $0 \leq d \leq 2|Q|$ in the rack case), but what other conditions are necessary or sufficient? Can we deduce from a quandle's polynomial whether the quandle is isomorphic to a conjugation quandle, an Alexander quandle, a symplectic quandle, or a direct product, semidirect product, or an abelian extension of these?

For which specialized quandle polynomial invariants is there a quandle 2-cocycle (see [3]) such that $\Phi_{q} p$ is equal to the quandle cocycle invariant? Do we gain any information by combining the two, perhaps by setting

$$
\Psi_{\chi, q p}(K)=\sum_{f \in \operatorname{Hom}(Q(K), T)} z^{q p_{\operatorname{Im}(f) \subset T}\left(s_{0}, t_{0}\right)} t^{C o(f)}
$$

where $C o(f)$ is the sum of the cocycle values at the crossings in $K$ (see [3])?

\section{References}

[1] J. S. Carter, A. Crans, M. Elhamdadi and M. Saito. Cohomology of Categorical SelfDistributivity. arXiv.org math.GT/0607417

[2] J. S. Carter, M. Elhamdadi, M. Graña, and M. Saito. Cocycle knot invariants from quandle modules and generalized quandle homology. Osaka J. Math. 42 (2005) 499-541.

[3] J. S. Carter, D. Jelsovsky, S. Kamada, L. Langford and M. Saito. Quandle cohomology and state-sum invariants of knotted curves and surfaces. Trans. Amer. Math. Soc. 355 (2003) 39473989.

[4] F. M. Dionísio and P. Lopes. Quandles at finite temperatures. II. J. Knot Theory Ramifications 12 (2003) 1041-1092.

[5] R. Fenn and C. Rourke. Racks and links in codimension two. J. Knot Theory Ramifications 1 (1992), 343-406.

[6] R. Henderson, T. Macedo and S. Nelson. Symbolic computation with finite quandles. J. Symbolic Comput. 41 (2006) 811-817.

[7] B. Ho and S. Nelson. Matrices and finite quandles. Homology Homotopy Appl. 7 (2005) 197-208.

[8] D. Joyce. A classifying invariant of knots, the knot quandle. J. Pure Appl. Algebra 23 (1982) $37-65$.

[9] E. A. Navas and S. Nelson. On Symplectic Quandles. In Preparation.

[10] S. Nelson. Classification of finite Alexander quandles. Top. Proc. 27 (2003) 245-258.

[11] S. Nelson and C-Y. Wong. On the orbit decompostion of finite quandles. J. Knot Theory Ramifications 15 (2006) 761-772.

[12] D. Yetter. Quandles and monodromy. J. Knot Theory Ramifications 12 (2003) 523-541. 


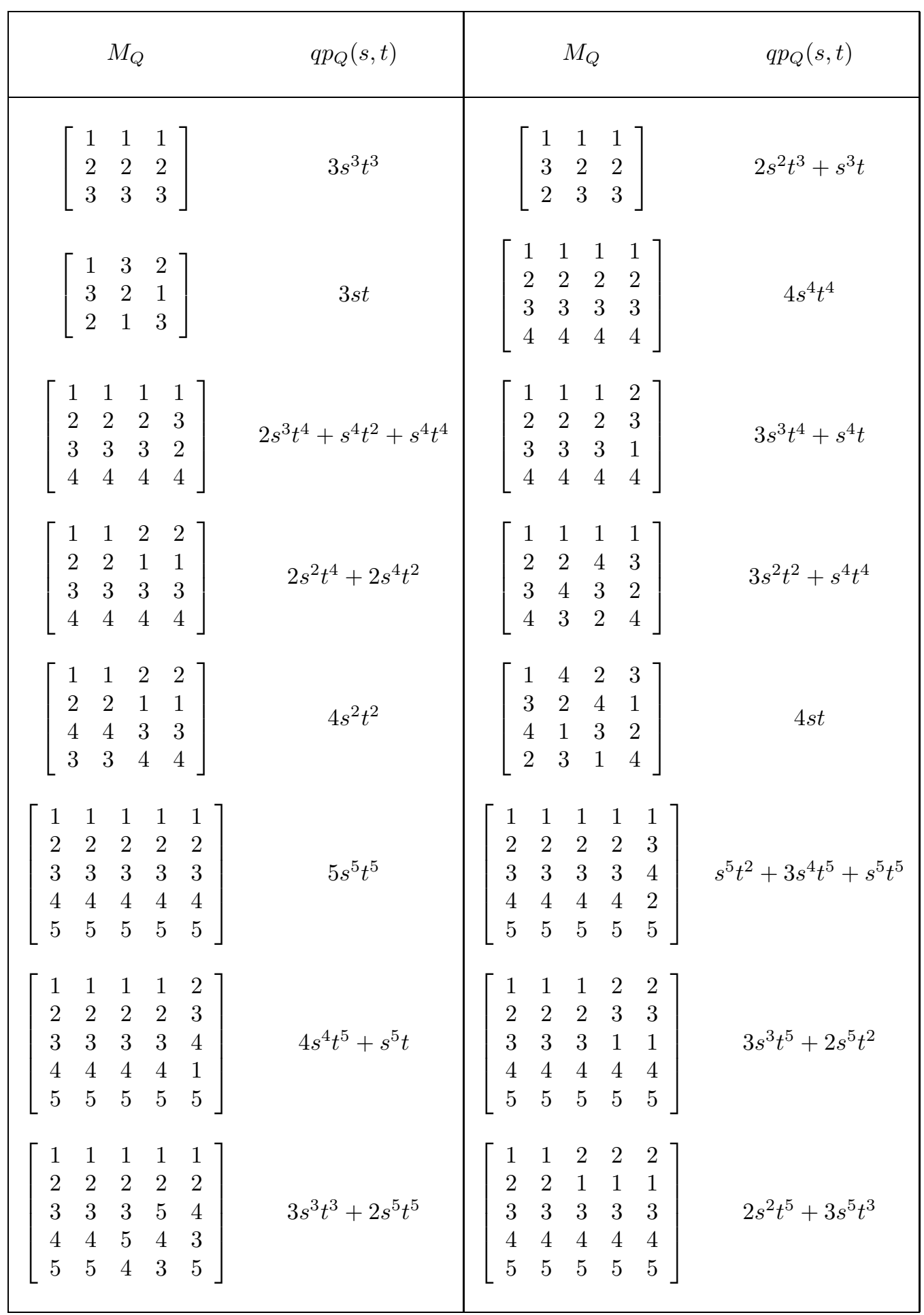

Table 1: $q p_{Q}(s, t)$ for quandles of order $\leq 5$ part I. 


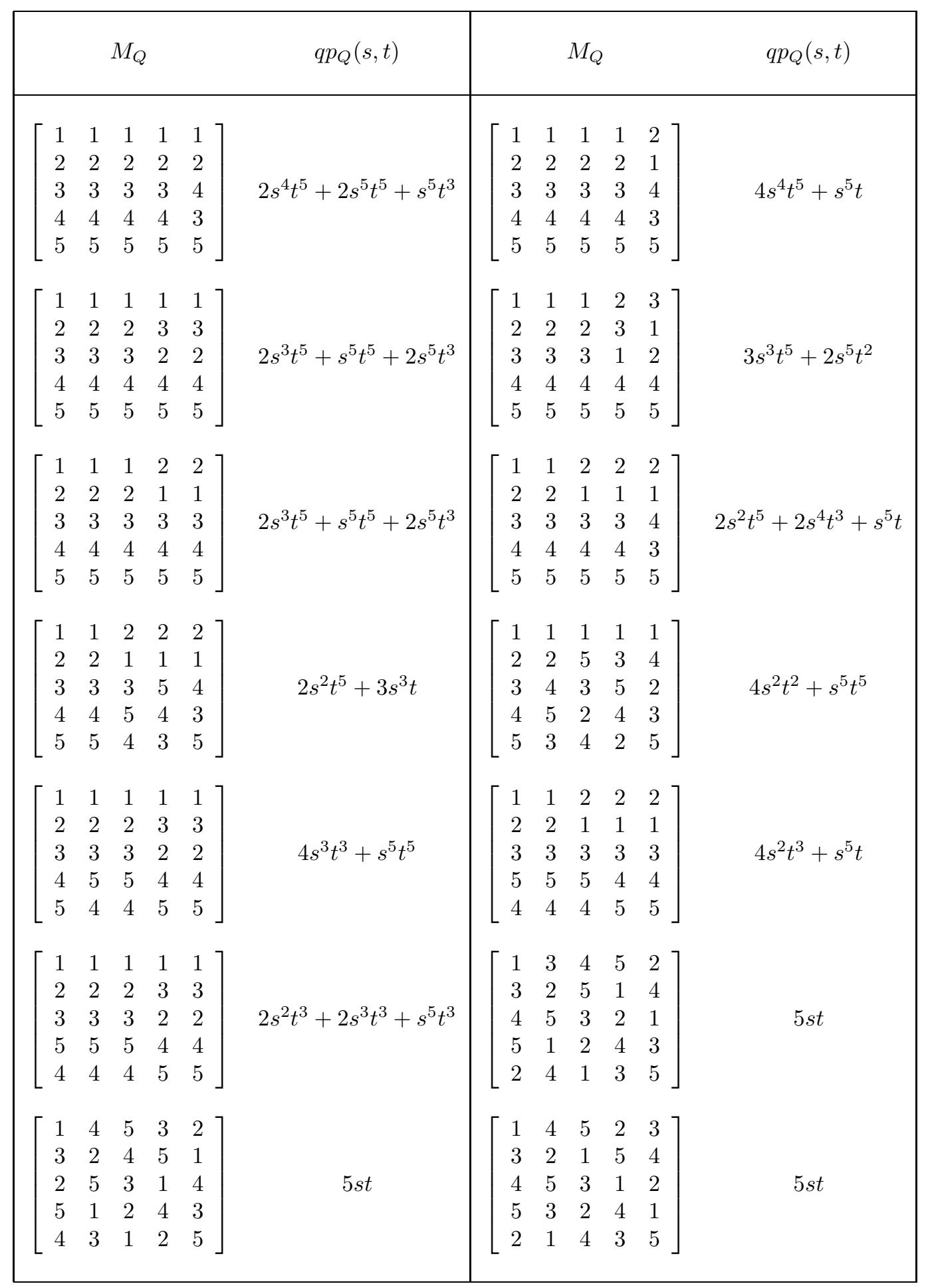

Table 2: $q p_{Q}(s, t)$ for quandles of order $\leq 5$ part II. 